\title{
A FISH study of chromosome fusion in the ICF syndrome: involvement of paracentric heterochromatin but not of the centromeres themselves
}

\author{
A T Sumner, A R Mitchell, P M Ellis
}

\begin{abstract}
We have used double fluorescence in situ hybridisation to study the involvement of centromeres and paracentromeric heterochromatin in the chromosome abnormalities seen in the ICF syndrome. To detect centromeres, we used a probe which labelled alphoid satellite DNA, and for the paracentromeric heterochromatin a probe for classical satellite II. Our results show that it is always the paracentromeric heterochromatin of the relevant chromosomes that becomes decondensed in this syndrome and which fuses to produce multiradial configurations. However, the centromeric regions, identified by their content of alphoid satellite DNA, appear never to become decondensed and always remain outside the regions of chromosome fusion in the multiradials. (于 Med Genet 1998;35:833-835)
\end{abstract}

Keywords: ICF syndrome; heterochromatin; centromeres; FISH

The ICF syndrome is a rare human genetic disorder, the symptoms of which include Immunodeficiency, Centromeric instability, and Facial anomalies. The centromeric instability includes decondensation of the heterochromatin of chromosomes 1,9 , and 16 , and, very occasionally, of one or two other chromosomes, and the fusion of the heterochromatic regions of these chromosomes to form multiradial configurations, comprising arms from several of these chromosomes. For recent reports on the chromosome abnormalities found in this syndrome, see Smeets et al, ${ }^{1}$ Brown $e t ~ a l,{ }^{2}$ and Schuffenhauer $e t a l$; the first two of these also review all the cases reported up to their dates of publication.

Although the large blocks of heterochromatin on chromosomes 1,9 , and 16 can become decondensed and may fuse to form multiradial configurations in patients with this syndrome, it has not been clear whether the centromeres themselves are involved in these processes. Using $C_{d}$ staining, Gimelli et al $l^{4}$ postulated that the centromeres themselves were not directly involved in the formation of multiradials, but that the paracentromeric heterochromatin, which was identified by in situ hybridisation, was involved. However, although $C_{d}$ staining is generally regarded as a marker for centromeres, the basis of its specificity is not known. ${ }^{5}$
Miniou $e t a l^{b}$ and Schuffenhauer $e t a l^{\beta}$ also used FISH to show the involvement of classical satellite II in the decondensation and fusion of chromosomes in the ICF syndrome, but did not show images of FISH studies of the centromeres themselves. We have therefore used two colour fluorescence PRINS in situ hybridisation (FISH) to study the centromeres and paracentromeric heterochromatin simultaneously in chromosomes from a patient with the ICF syndrome.

\section{Materials and methods}

The patient from whom chromosomes were obtained for this study has already been described in detail, ${ }^{2}$ and chromosome preparations were obtained from the patient as described in that paper. Fluorescence in situ hybridisation was carried out using a double PRINS technique, ${ }^{7}$ described in detail by Mitchell et al. ${ }^{8}$ Two oligonucleotides were used. Oligonucleotide 405 detects alphoid repetitive DNA sequences, ${ }^{9}$ while B18 hybridises to the human satellite II simple sequence family. The sequence of $\mathrm{B} 18$ is 5'CGTTGATTCCATTTGATGTTGATTCCA TTC- $3{ }^{\prime}{ }^{10}$ Oligonucleotide 405 incorporated digoxigenin-1 1-dUTP during the PRINS reaction, and the digoxygenin was detected using anti-digoxygenin-FITC FAB fragments (Boehringer Mannheim). The oligonucleotide B18 incorporated bio-11-dUTP (Boehringer Mannheim) during the PRINS reaction, and detection of the biotin was achieved using avidinTexas red (Vector Laboratories). Chromosomes were labelled with DAPI to produce blue fluorescence. The images were captured using a Zeiss Axioplan fluorescence microscope equipped with a $100 \mathrm{~W}$ mercury lamp and a photometric CCD camera. Images were captured and processed using software from Digital Scientific (Cambridge, England). Exact registration of images was achieved using a Chroma P1 filter set mounted on a rotating wheel. False colour images were photographed from the computer screen; the colours were chosen to correspond as closely as possible to the natural colours of the fluorescence, so that DAPI appears blue on the photographs, sites of alphoid satellite DNA green, and satellite II pink.

\section{Results}

The probe for alphoid satellite DNA, oligonucleotide 405 , labelled all or most of the centromeres in every metaphase examined, whereas 

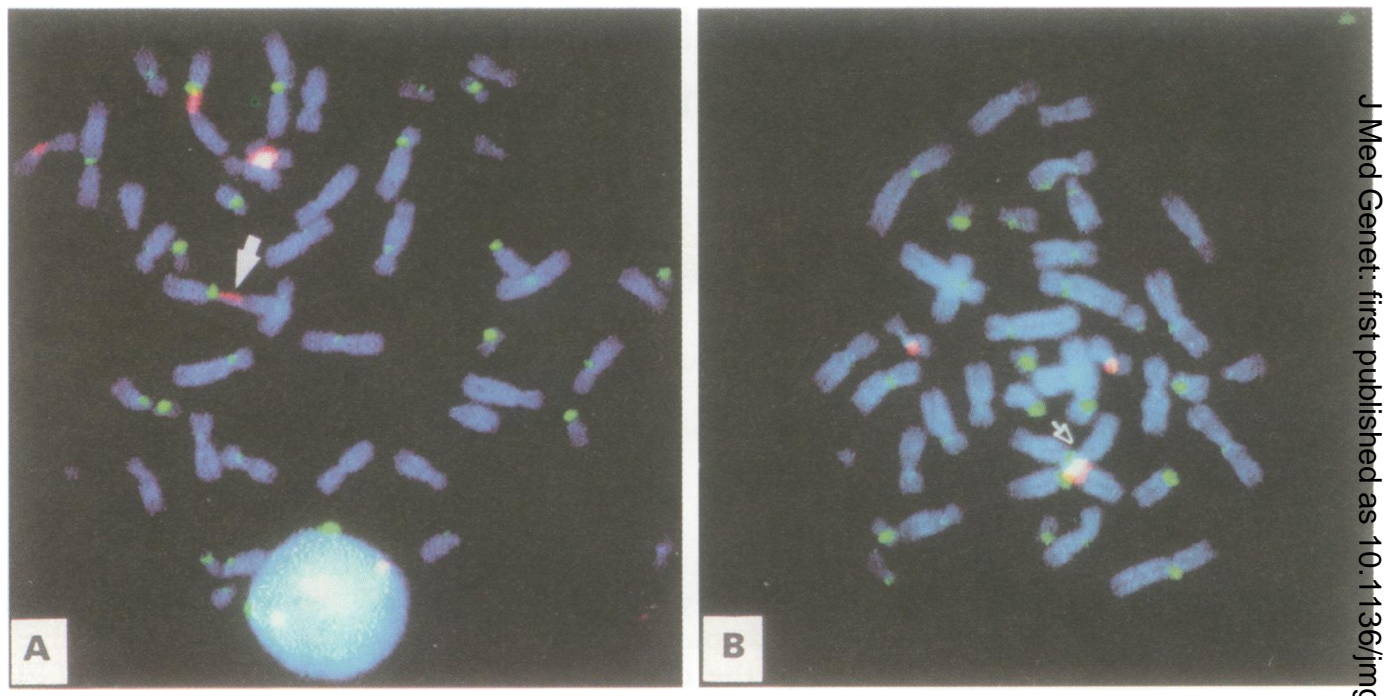

Figure 1 Metaphase spreads from the patient with ICF syndrome showing results of double in situ hybridisation with the $\dot{\omega}_{\mathrm{w}}$ probe for alphoid satellite DNA (green) and the probe for satellite II (pink). Chromosomes are counterstained with DAPI G (blue). (A) Extended heterochromatin is labelled pink for satellite II (arrow), while the green centromeric signal remains $\vec{\circ}$ compact. (B) Multiradial configuration (arrowed) showing a single fused block of heterochromatin labelled pink (satellite $i_{\infty}$ II), surrounded by separate green signals for the centromeres (alphoid satellite). Note that the very pale pink fluorescence ifw the multiradial configuration is the result of overexposure of the photograph in this region of highly concentrated satellite DNA.

the satellite II probe, B18, labelled only the paracentromeric heterochromatin of chromosomes 1,9 , and 16 (fig 1). However, when the heterochromatin was decondensed, the thin thread produced label throughout its length with the satellite II probe, while the region labelled with the alphoid satellite probe remained condensed, and showed the same appearance as in chromosomes that did not show any decondensation of the heterochromatin (fig 1A).

In multiradial configurations, the region labelled with the satellite II probe always formed a single block (fig 1B), while the alphoid satellite probe labelled discrete blocks on individual chromosome arms surrounding the block of satellite II. The multiradials are, therefore, formed by fusion of the paracentromeric heterochromatin, while the centromeres themselves remain separate.

\section{Discussion}

In this study, we have used oligonucleotide probes which recognise alphoid satellite DNA and classical satellite II DNA to label the centromeres and the paracentromeric heterochromatin, respectively, in chromosomes from a patient with the ICF syndrome. As shown by Mitchell et al for alphoid and satellite III DNA in man, the two families of repetitive DNA studied here also occupy distinct domains. Our results confirm that it is the regions of paracentromeric heterochromatin, containing satellite II, which can become decondensed in this syndrome and can fuse to form multiradials. This is in agreement with morphological studies that pointed to the decondensation and fusion of heterochromatin (see, in particular, the scanning electron microscopy studies reported by Brown et $a l^{2}$ ), and confirms more recent FISH studies. ${ }^{34611}$ It is equally clear that the centromeres, as identified by hybridisation of the alphoid satellite probe, do not appear to decondense and are excluded from the fusions by which the multiradials are formed. This is, of course, consistent with the observation that normally only those chromo somes which carry substantial blocks of paracentromeric heterochromatin are involve in the chromosome abnormalities characteristic of the ICF syndrome.

It is not, at present, possible to explain full the connection between the various features of the ICF syndrome, but it seems plausible thif the same process could give rise to the immut nodeficiency and the formation of multiradia chromosomes. Both immunodeficiency and the production of multiradials could be the result of a failed recombination process. It has: been shown that recombination can occu between experimentally decondensed seg் ments of heterochromatin. ${ }^{12}$ The process in volved in the ICF syndrome would presumab be failure to resolve recombination intermedi ates rather than failure to initiate recombin tion, otherwise no multiradials would bs formed.

1 Smeets DFCM, Moog U, Weemaes CMR, et al. ICP syndrome: a new case and review of the literature. Hup Genet 1994;94:240-6.

2 Brown DC, Grace E, Sumner AT, Edmunds AT, Ellis PN. ICF syndrome (immunodeficiency, centromeric instability and facial anomalies): investigation of heterochromatim and facial anomalies): investigation of heterochromatem abnormalities and review of clinical outcome. Hum Genest
1995;96:411-16.

3 Schuffenhauer S, Bartsch O, Stumm M, et al. DNA, FISH and complementation studies in ICF syndrome: DNA hypomethylation of repetitive and single copy loci and exp dence for a trans acting factor. Hum Genet 1995;96:562-7\$

4 Gimelli G, Varone P, Pezzolo A, Lerone M, Pistoia V. ICFF syndrome with variable expression in sibs. $\mathcal{F}$ Med Gerif 1993;30:429-32.

5 Sumner AT. Chromosome banding. London: Unwin Hyma 1990.

6 Miniou P, Jeanpierre M, Blanquet V, et al. Abnormal met\$ ylation pattern in constitutive and facultative $(X$ inactif chromosome) heterochromatin of ICF patients. Hum MO Genet 1994;3:2093-102.

7 Koch JE, Kølvraa S, Petersen KB, Gregersen N, Bolund Oligonucleotide priming methods for the chromosomespecific labelling of alpha satellite DNA in situ. Chromosoma 1989;98:259-65.

8 Mitchell AR, Nicol L, Malloy P, Kipling D. Novel structural organisation of a Mus musculus DBA/2 chromosome shows 85 . 
9 Mitchell A, Jeppesen P, Hanratty D, Gosden J. The organization of repetitive DNA sequences on human chromosomes with respect to the kinetochore analyzed using a combination of oligonucleotide primers and CREST anticentromere serum. Chromosoma 1992;101:333-41.

10 Hollis M, Hindley J. Satellite II DNA of human lymphocytes: tandem repeats of a simple sequence element. Nucleic Acids Res 1988;116:363.

11 Stacey M, Bennett MS, Hulten M. FISH analysis on spontaneously arising micronuclei in the ICF syndrome. $\mathcal{F} M e d$ Genet 1995;32:502-8.

12 Kokalj-Vokac N, Almeida A, Viegas-Péquignot E, Jeanpierre $M$, Malfoy B. Dutrillaux B. Specific induction of uncoiling and recombination by azacytidine in classical satellitecontaining constitutive heterochromatin. Cytogenet Cell Genet 1993;63:11-15. 